\title{
PERFIL, FATORES DE RISCO E PREVALÊNCIA DA VIOLÊNCIA CONTRA A MULHER
}

PROFILE, RISK FACTORS, AND PREVALENCE OF VIOLENCE AGAINST WOMEN

PERFIL, FACTORES DE RIESGO Y PREVALENCIA DE LA VIOLENCIA CONTRA LA MUJER

Letícia Barbosa Dias ${ }^{1}$

Lisie Alende Prates 2

Luiza Cremonese ${ }^{3}$

\section{Como Citar:}

Dias LB, Prates LA, Cremonese L. Perfil, fatores de risco e prevalência da violência contra a mulher. Sanare

(Sobral, Online). 2021;20(1):102-

Palavras-chave:

Saúde da mulher; Mulheres; Gênero e saúde; Violência; Violência contra a mulher.

Keywords:

Women's health; Women; Gender and health; Violence; Violence against women.

\section{Palabras clave:}

Salud de la mujer; Mujeres; Género y salud; Violencia; La violencia contra la mujer.

Submetido: $08 / 01 / 2021$

Aprovado: $17 / 06 / 2021$

Autor(a) para Correspondência: Lisie Alende Prates E-mail: lisieprates@unipampa.edu.br

\section{RESUMO}

Este estudo objetiva analisar as evidências disponíveis na literatura acerca do perfil, fatores de risco e prevalência da violência contra a mulher. Trata-se de revisão integrativa realizada na BDENF, LILACS, PubMed e Scopus. Foram incluídos artigos de estudos primários, realizados no Brasil e disponíveis em português. A partir da análise dos 26 artigos incluídos, verificou-se que a violência contra a mulher prevalece entre mulheres jovens; pretas, negras ou pardas; católicas; casadas ou que conviviam com parceiros intimos; mães ou que estavam grávidas e não exerciam atividade remunerada. 0 uso de drogas elou álcool pela mulher ou agressor, baixa escolaridade, condição socioeconômica vulnerável, histórico de violência intrafamiliar na infância, iniciação sexual precoce, infância ausente de brincadeiras e afeto, falta de suporte familiar e dependência financeira do agressor emergiram como fatores de risco. Esses achados podem auxiliar no desenvolvimento de intervenções para o enfrentamento da violência contra a mulher.

1.Discente do curso de graduação em Enfermagem. Universidade Federal do Pampa (UNIPAMPA). Uruguaiana, Rio Grande do Sul (RS), Brasil. E-mail: bdiasleticia@gmail.com; ORCID: https://orcid.org/0000-0002-1859-9707

2. Enfermeira. Doutora em Enfermagem. Docente da UNIPAMPA. Uruguaiana, RS, Brasil. E-mail: lisieprates@ unipampa.edu.br; ORCID: http://orcid.org/0000-0002-5151-0292

3. Enfermeira. Doutora em Enfermagem. Docente da Universidade Luterana do Brasil. Cachoeira do Sul, RS, Brasil. E-mail: lu_cremonese@hotmail.com; 0RCID: https://orcid.org/0000-0001-7169-1644

Cert. de Redação Científica: Central das Revisões. Edição de texto: Karina Maria M. Machado. Revisão de provas: Texto definitivo validado pelos(as) autores(as). 


\section{ABSTRACT}

This study aims to analyze the evidence available in the literature about the profile, risk factors, and prevalence of violence against women. This is an integrative review carried out at BDENF, LILACS, PubMed, and Scopus. Articles from primary studies conducted in Brazil and available in Portuguese were included. From the analysis of the 26 articles included, we found that violence against women is prevalent among young women; black or brown; Catholic; married or living with intimate partners; mothers or who were pregnant and did not have a paid job. The use of drugs and/or alcohol by the woman or aggressor, low education, vulnerable socioeconomic status, history of intrafamily violence during childhood, early sexual initiation, childhood without playful games and affection, lack of family support and financial dependence of the aggressor emerged as factors of risk. These findings can, therefore, help in the development of interventions to fight violence against women.

\section{RESUMEN}

Este estudio tiene como objetivo analizar la evidencia disponible en la literatura sobre el perfil, los factores de riesgo y la prevalencia de la violencia contra la mujer. Se trata de una revisión integradora realizada en BDENF, LILACS, PubMed y Scopus. Se incluyeron artículos de estudios primarios realizados en Brasil y disponibles en portugués. Del análisis de los 26 artículos incluidos, se encontró que la violencia contra la mujer prevalece entre las mujeres jóvenes; negras; católicas; casadas o que viven con parejas íntimas; madres o que estaban embarazadas y no ejercían un trabajo remunerado. Surgió el uso de drogas y/o alcohol por parte de la mujer o agresor, baja escolaridad, condición socioeconómica vulnerable, antecedentes de violencia intrafamiliar en la niñez, iniciación sexual precoz, niñez ausente de juegos y afecto, ausencia de apoyo familiar y dependencia económica del agresor como factores de riesgo. Estos hallazgos pueden, por lo tanto, ayudar en el desarrollo de intervenciones para combatir la violencia contra la mujer.

\section{INTRODUÇÃO}

A violência pode ser definida pelo uso intencional de força física ou poder, real ou em ameaça, que resulte em danos físicos ou psicológicos, morte ou privaçã $0^{1}$. Nesse contexto, tem-se a violência contra a mulher (VCM), considerada como um problema de saúde pública, que requer ações conjuntas entre os órgãos governamentais, civis e instituições².

A VCM é constituída por um ciclo, que inicia de maneira lenta e quase imperceptível, podendo atingir dimensões perigosas e desafiadoras. Esse ciclo é composto por três fases: a primeira consiste na tensão no relacionamento, caracterizada por conflitos leves; a segunda revela o episódio agudo de violência e é marcada pelas agressões; já a terceira é chamada de lua de mel, em que há o arrependimento e remorso do agressor, além do medo da separação ${ }^{3}$.

Segundo dados do Relatório Mundial da Saúde, $10 \%$ a $69 \%$ das mulheres do mundo todo relataram ter sofrido agressão física por parceiro íntimo em alguma ocasião da vida ${ }^{1}$. No Brasil, estima-se que a VCM cause mais mortes às mulheres de 15 a 44 anos que o câncer, a malária, os acidentes de trânsito e as guerras ${ }^{4}$.

Nesse contexto, é preciso salientar que a VCM gera traumas físicos e emocionais consideráveis ${ }^{4}$. As consequências psicológicas constituem um sério agravo à saúde da mulher, que, mesmo após o rompimento do ciclo da violência, pode desenvolver transtorno de estresse pós-traumático e depressão ${ }^{5}$.

A partir disso, reconhece-se a necessidade de estudos que tenham a VCM como objeto de investigação, tal como propõe a Agenda Nacional de Prioridades de Pesquisa em Saúde ${ }^{6}$. Assim, este estudo teve como objetivo analisar as evidências disponiveis na literatura brasileira acerca do perfil, fatores de risco e prevalência da violência contra a mulher.

\section{METODOLOGIA}

Estudo de revisão integrativa, desenvolvido em junho de 2020, percorrendo as seguintes etapas: 1) Formulação da pergunta; 2) Amostragem; 3) Extração de dados dos estudos primários; 4) 
Avaliação crítica; 5) Análise e síntese dos resultados da revisão; e 6) Apresentação da revisão ${ }^{7}$. A questão guia foi: qual é o perfil, fatores de risco e prevalência da violência contra a mulher nos estudos disponíveis na literatura científica brasileira?

As bases de dados consultadas foram a Literatura Latino-Americana e do Caribe em Ciências da Saúde (LILACS); Banco de Dados em Enfermagem (BDENF) via Biblioteca Virtual em Saúde (BVS); National Library of Medicine (PubMed) e SciVerse (Scopus) via Portal de Periódicos Capes. Respeitaram-se as variações existentes nas buscas em cada base e, por isso, foram necessárias algumas alterações nas estratégias (Figura 1).

Quadro 1. Estratégias de busca de acordo com as bases de dados utilizadas.

\begin{tabular}{|c|c|}
\hline Bas & sca \\
\hline $\begin{array}{l}\text { LILACS } \\
\text { BDENF } \\
\text { via BVS }\end{array}$ & $\begin{array}{l}\text { tw:((tw:((mh:((saúde da mulher))) OR (mh:(mulheres)) OR (mh:((gênero e saúde))) )) } \\
\text { AND (tw:( (mh:(violência })) \text { OR (mh:((violência contra a mulher }))) \text { OR (mh:((violência } \\
\text { por parceiro íntimo))) OR (mh:((violência doméstica))) OR (mh:((violência sexual))) } \\
\text { OR (mh:((delitos sexuais))) OR (mh:((violência de gênero }))))) \text { AND ( db:(“LILACS" OR } \\
\text { "BDENF") AND la:(“pt")) }\end{array}$ \\
\hline PubMed & 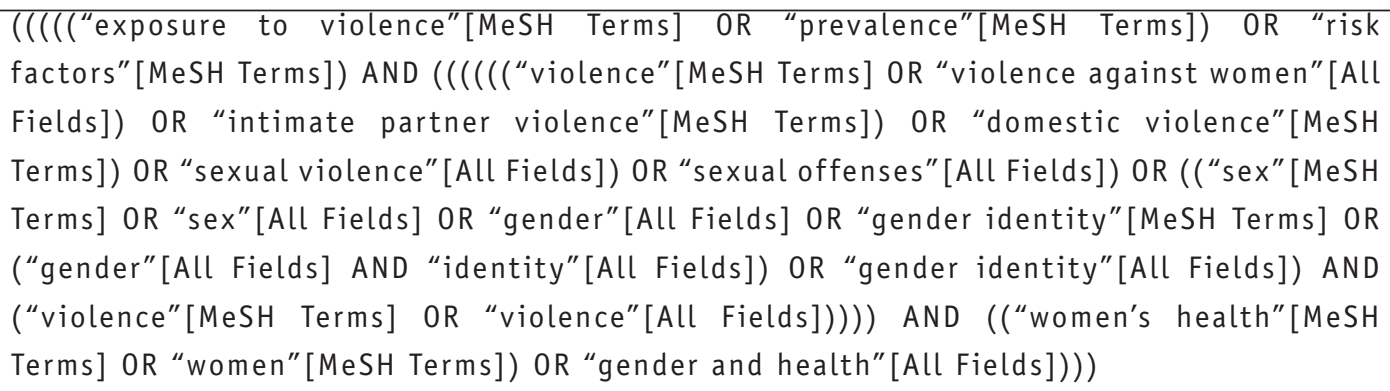 \\
\hline Scopus & $\begin{array}{l}\text { ((KEY ("Women's health") OR TITLE-ABS-KEY ("Women") OR KEY ("Gender and health"))) } \\
\text { AND (( KEY (“Violence") OR KEY ("Violence against women") OR TITLE-ABS-KEY (“Intimate } \\
\text { partner violence") OR KEY (“Domestic violence") OR KEY (sexual AND violence) OR TITLE- } \\
\text { ABS-KEY (sexual AND offenses) OR KEY (gender AND violence))) AND ((KEY ("Exposure } \\
\text { to Violence") OR KEY ("Prevalence") OR KEY (“Risk factors"))) AND (LIMIT- } \\
\text { TO (LANGUAGE, "Portuguese")) }\end{array}$ \\
\hline
\end{tabular}

Fonte: dados da pesquisa.

Os critérios de inclusão foram: artigos da temática, oriundos de pesquisas primárias, disponíveis online, em português, realizados no Brasil. 0s estudos identificados de forma duplicada entre as bases foram contabilizados apenas uma vez. A elegibilidade das publicações foi desenvolvida a partir da concordância de duas revisoras.

Após a busca, procedeu-se à leitura dos seus títulos e resumos. Assim, 26 artigos compuseram o corpus de análise, sendo três na BVS, seis na PubMed e 17 na Scopus (Figura 2). 
Figura 2. Fluxograma do processo de identificação, seleção, elegibilidade e inclusão do estudo.

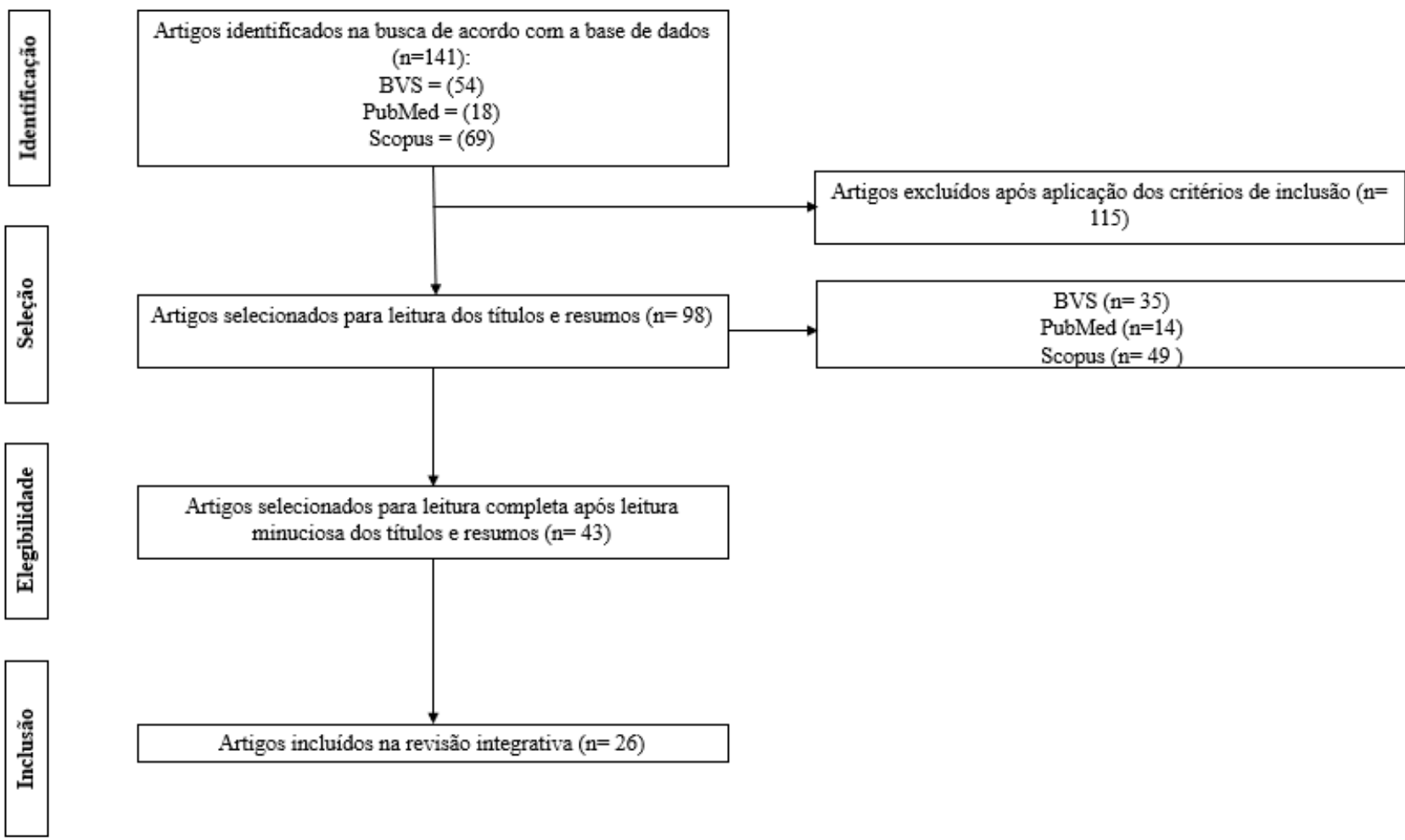

Fonte: dados da pesquisa.

Por fim, realizou-se a análise e síntese dos resultados da revisão, o que permitiu identificar os achados que responderam à questão guia. A seguir, apresentam-se as evidências científicas disponíveis sobre o objeto de pesquisa.

\section{RESULTADOS}

Para a análise e a organização das evidências, utilizou-se um quadro sinóptico elaborado pelas autoras para a extração dos dados dos estudos. Também foi realizada a avaliação crítica dos estudos incluídos, abrangendo a análise dos níveis de evidência.

Quadro 1 - Detalhamento dos artigos incluídos na revisão integrativa.

\begin{tabular}{|c|c|c|c|c|}
\hline ID & TÍTULO & OBJETIVO & MÉTODO & NE \\
\hline $\mathrm{A} 1^{8}$ & $\begin{array}{l}\text { Violência contra mulheres } \\
\text { na perspectiva dos } \\
\text { agentes comunitários de } \\
\text { saúde. }\end{array}$ & $\begin{array}{c}\text { Conhecer e compreender a violência } \\
\text { contra as mulheres na perspectiva } \\
\text { dos agentes comunitários de saúde } \\
\text { inseridos nas Estratégias de Saúde } \\
\text { da Família (ESF) de um município da } \\
\text { região noroeste do Rio Grande do } \\
\text { Sul. }\end{array}$ & $\begin{array}{l}\text { Qualitativo, } \\
\text { exploratório e } \\
\text { descritivo. }\end{array}$ & $2^{*}$ \\
\hline $\mathrm{A} 2^{9}$ & $\begin{array}{l}\text { Violência doméstica na } \\
\text { gravidez: prevalência e } \\
\text { fatores associados. }\end{array}$ & $\begin{array}{l}\text { Identificar os fatores associados } \\
\text { à violência doméstica contra } \\
\text { gestantes. }\end{array}$ & $\begin{array}{l}\text { Misto, com análise } \\
\text { de corte transversal, } \\
\text { seguido de estudo de } \\
\text { coorte. }\end{array}$ & $4^{*}$ \\
\hline
\end{tabular}




\begin{tabular}{|c|c|c|c|c|}
\hline ID & TÍTULO & OBJETIVO & MÉTODO & NE \\
\hline $\mathrm{A} 3^{10}$ & $\begin{array}{l}\text { Violência física grave } \\
\text { entre parceiros íntimos } \\
\text { como fator de risco para a } \\
\text { inadequação no rastreio do } \\
\text { câncer de colo de útero. }\end{array}$ & $\begin{array}{l}\text { Avaliar a violência física grave entre } \\
\text { parceiros íntimos como fator de } \\
\text { risco para a inadequação no rastreio } \\
\text { do câncer do colo do útero no } \\
\text { âmbito da ESF. }\end{array}$ & Caso-controle. & 4 * \\
\hline $\mathrm{A} 4^{11}$ & $\begin{array}{c}\text { A violência na vida de } \\
\text { mulheres em situação } \\
\text { de rua na cidade de São } \\
\text { Paulo, Brasil. }\end{array}$ & $\begin{array}{l}\text { Trazer à reflexão situações de } \\
\text { violência na vida de mulheres em } \\
\text { condição de rua, quiçá subsidiar } \\
\text { a crítica sobre esta questão, que } \\
\text { engloba três grandes temas que, } \\
\text { quando juntos, evidenciam a } \\
\text { desigualdade social: mulheres, sem- } \\
\text { teto, violência. }\end{array}$ & Cartografia. & 2 * \\
\hline$A 5^{12}$ & $\begin{array}{l}\text { MARIA, MARIA: concepções } \\
\text { sobre ser mulher em } \\
\text { situação de rua. }\end{array}$ & $\begin{array}{c}\text { Conhecer quais são as concepções } \\
\text { sobre ser mulher nesse contexto, a } \\
\text { partir do depoimento de mulheres } \\
\text { em situação de rua, e assim, dar a } \\
\text { necessária visibilidade para essa } \\
\text { situação. }\end{array}$ & $\begin{array}{l}\text { Qualitativo, } \\
\text { exploratório e } \\
\text { descritivo. }\end{array}$ & 2 * \\
\hline$A 6^{13}$ & $\begin{array}{l}\text { Gênero, violência e viver } \\
\text { na rua: vivências de } \\
\text { mulheres que fazem uso } \\
\text { problemático de drogas. }\end{array}$ & $\begin{array}{c}\text { Descrever vivências de mulheres, } \\
\text { em situação de rua, que fazem uso } \\
\text { problemático de drogas. }\end{array}$ & Qualitativo. & 2 * \\
\hline$A 7^{14}$ & $\begin{array}{l}\text { Prevalência da violência } \\
\text { contra a mulher usuária de } \\
\text { serviço de saúde. }\end{array}$ & $\begin{array}{c}\text { Determinar a prevalência desse tipo } \\
\text { de violência entre as usuárias de um } \\
\text { centro de saúde distrital. }\end{array}$ & $\begin{array}{c}\text { Quantitativo e } \\
\text { transversal. }\end{array}$ & $4^{*}$ \\
\hline$A 8^{15}$ & $\begin{array}{l}\text { Violências contra } \\
\text { mulheres por parceiro } \\
\text { íntimo em área urbana } \\
\text { economicamente } \\
\text { vulnerável, Brasília, DF. }\end{array}$ & $\begin{array}{l}\text { Estimar a prevalência de tipos de } \\
\text { violência e de comportamentos de } \\
\text { controle praticados por parceiros } \\
\text { intimos contra mulheres residentes } \\
\text { em área economicamente vulnerável. }\end{array}$ & Transversal. & $4^{*}$ \\
\hline$A 9^{16}$ & $\begin{array}{l}\text { Violência contra mulheres: } \\
\text { a experiência de usuárias } \\
\text { de um serviço de urgência } \\
\text { e emergência de Salvador, } \\
\text { Bahia, Brasil. }\end{array}$ & $\begin{array}{l}\text { Investigar a ocorrência de violência } \\
\text { - física, sexual e psicológica } \\
\text { - entre usuárias de um serviço de } \\
\text { urgência e emergência e a sua } \\
\text { distribuição segundo características } \\
\text { sociodemográficas. }\end{array}$ & Corte transversal. & $4^{*}$ \\
\hline $\mathrm{A} 10^{17}$ & $\begin{array}{l}\text { Prevalência e fatores } \\
\text { associados à violência } \\
\text { sofrida em mulheres } \\
\text { encarceradas por tráfico } \\
\text { de drogas no Estado de } \\
\text { Pernambuco, Brasil: um } \\
\text { estudo transversal. }\end{array}$ & $\begin{array}{l}\text { Estimar a prevalência e os fatores } \\
\text { associados à violência física, } \\
\text { psicológica e sexual sofrida por } \\
\text { mulheres encarceradas pelo tráfico } \\
\text { de drogas nos } 24 \text { meses anteriores ao } \\
\text { encarceramento numa penitenciária } \\
\text { do Estado de Pernambuco. }\end{array}$ & $\begin{array}{l}\text { Observacional, } \\
\text { descritivo, do tipo } \\
\text { corte transversal. }\end{array}$ & $4^{*}$ \\
\hline
\end{tabular}




\begin{tabular}{|c|c|c|c|c|}
\hline ID & TÍTULO & OBJETIVO & MÉTODO & NE \\
\hline $\mathrm{A} 11^{18}$ & $\begin{array}{l}\text { Perfil da violência de } \\
\text { gênero perpetrada por } \\
\text { companheiro. }\end{array}$ & $\begin{array}{l}\text { Verificar a prevalência e o perfil } \\
\text { da violência de gênero (física, } \\
\text { psicológica e sexual) perpetrada } \\
\text { contra a mulher pelo parceiro(a) } \\
\text { atual ou passado. }\end{array}$ & Transversal. & $4^{*}$ \\
\hline $\mathrm{A} 12^{19}$ & $\begin{array}{l}\text { HIV e violência contra } \\
\text { mulheres: estudo em } \\
\text { município com alta } \\
\text { prevalência de Aids no Sul } \\
\text { do Brasil. }\end{array}$ & $\begin{array}{l}\text { Investigar a prevalência e os fatores } \\
\text { associados à violência contra } \\
\text { mulheres com HIV em um município } \\
\text { de porte médio no Sul do Brasil. }\end{array}$ & Transversal. & $4^{*}$ \\
\hline $\mathrm{A} 13^{20}$ & $\begin{array}{c}\text { Violência contra a mulher } \\
\text { em Vitória, Espírito Santo, } \\
\text { Brasil. }\end{array}$ & $\begin{array}{l}\text { Estimar a prevalência e os fatores } \\
\text { associados às violências psicológica, } \\
\text { física e sexual nas mulheres } \\
\text { vítimas de violência perpetrada } \\
\text { pelo parceiro íntimo atendidas nos } \\
\text { serviços de atenção primária. }\end{array}$ & Transversal. & $4^{*}$ \\
\hline $\mathrm{A} 14^{21}$ & $\begin{array}{c}\text { Violência por parceiro } \\
\text { íntimo e prática educativa } \\
\text { materna. }\end{array}$ & $\begin{array}{l}\text { Analisar a associação entre a } \\
\text { violência pelo parceiro íntimo contra } \\
\text { a mulher e a prática educativa } \\
\text { materna direcionada às crianças no } \\
\text { início da escolaridade formal. }\end{array}$ & Transversal. & $4^{*}$ \\
\hline $\mathrm{A} 15^{22}$ & $\begin{array}{l}\text { Violência conjugal física } \\
\text { contra a mulher na vida: } \\
\text { prevalência e impacto } \\
\text { imediato na saúde, } \\
\text { trabalho e família. }\end{array}$ & $\begin{array}{l}\text { Estimar a prevalência de violência } \\
\text { conjugal física contra a mulher } \\
\text { ao longo da vida (VCFM) em uma } \\
\text { comunidade urbana de baixa renda } \\
\text { e avaliar o seu impacto imediato na } \\
\text { saúde, trabalho e vida familiar. }\end{array}$ & Coorte transversal. & $4^{*}$ \\
\hline $\mathrm{A} 16^{23}$ & $\begin{array}{c}\text { Prevalência e procura } \\
\text { de ajuda na violência } \\
\text { conjugal física ao longo } \\
\text { da vida. }\end{array}$ & $\begin{array}{l}\text { Estimar a prevalência de violência } \\
\text { conjugal física ao longo da vida em } \\
\text { mulheres de comunidade urbana de } \\
\text { baixa renda e identificar os tipos de } \\
\text { ajuda procurados pelas vítimas. }\end{array}$ & $\begin{array}{c}\text { Estudo-piloto brasileiro } \\
\text { de corte transversal. }\end{array}$ & $4^{*}$ \\
\hline $\mathrm{A} 17^{24}$ & $\begin{array}{l}\text { Violência entre parceiros } \\
\text { intimos e álcool: } \\
\text { prevalência e fatores } \\
\text { associados. }\end{array}$ & $\begin{array}{l}\text { Estimar a violência física entre } \\
\text { parceiros íntimos e examinar a } \\
\text { associação entre a violência e } \\
\text { variáveis sociodemográficas, uso de } \\
\text { álcool e outros fatores relacionados. }\end{array}$ & $\begin{array}{c}\text { Inquérito } \\
\text { epidemiológico. }\end{array}$ & $4^{*}$ \\
\hline $\mathrm{A} 18^{25}$ & $\begin{array}{c}\text { Qualidade de vida e } \\
\text { depressão em mulheres } \\
\text { vítimas de seus parceiros. }\end{array}$ & $\begin{array}{c}\text { Avaliar a qualidade de vida e } \\
\text { depressão nas mulheres vítimas da } \\
\text { violência doméstica; estabelecer } \\
\text { o perfil socioeconômico da } \\
\text { mulher agredida pelo parceiro e } \\
\text { as particularidades das agressões } \\
\text { sofridas. }\end{array}$ & Quantitativo. & $4^{*}$ \\
\hline
\end{tabular}




\begin{tabular}{|c|c|c|c|c|}
\hline ID & TÍTULO & OBJETIVO & MÉTODO & NE \\
\hline $\mathrm{A} 19^{26}$ & $\begin{array}{c}\text { Tentativa de suicídio, } \\
\text { transtorno de estresse } \\
\text { pós-traumático e fatores } \\
\text { associados em mulheres do } \\
\text { Recife. }\end{array}$ & $\begin{array}{l}\text { Investigar a associação da tentativa } \\
\text { de suicídio (TS) com o transtorno de } \\
\text { estresse pós-traumático (TEPT), a } \\
\text { violência por parceiro íntimo (VPI) e } \\
\text { variáveis relacionadas aos aspectos } \\
\text { socioeconômicos e demográficos em } \\
\text { uma coorte de mulheres cadastradas } \\
\text { na Estratégia Saúde da Família do } \\
\text { Recife. }\end{array}$ & Transversal. & $4^{*}$ \\
\hline $\mathrm{A} 20^{27}$ & $\begin{array}{l}\text { Estudo transversal sobre } \\
\text { fatores de risco de } \\
\text { violência por parceiro } \\
\text { intimo entre enfermeiras. }\end{array}$ & $\begin{array}{c}\text { Identificar os fatores de risco de } \\
\text { abuso por parceiro intimo entre } \\
\text { enfermeiras. }\end{array}$ & Transversal. & $4^{*}$ \\
\hline $\mathrm{A} 21^{28}$ & $\begin{array}{l}\text { Violência por parceiro } \\
\text { intimo entre puérperas: } \\
\text { fatores associados. }\end{array}$ & $\begin{array}{l}\text { Identificar o perfil e a autoestima } \\
\text { de puérperas, bem como as } \\
\text { características de seus bebês e } \\
\text { companheiros, e verificar suas } \\
\text { associações com a ocorrência de } \\
\text { Violência por Parceiro Íntimo (VPI). }\end{array}$ & Transversal. & $4^{*}$ \\
\hline $\mathrm{A} 22^{29}$ & $\begin{array}{c}\text { Domínios dos transtornos } \\
\text { mentais comuns em } \\
\text { mulheres que relatam } \\
\text { violência por parceiro } \\
\text { intimo. }\end{array}$ & $\begin{array}{l}\text { Verificar associações entre os tipos } \\
\text { de violência por parceiro íntimo e } \\
\text { os domínios dos transtornos mentais } \\
\text { comuns em mulheres. }\end{array}$ & Transversal. & $4^{*}$ \\
\hline $\mathrm{A} 23^{30}$ & $\begin{array}{l}\text { Sob a sombra da } \\
\text { maternidade: gravidez, } \\
\text { ideação suicida e violência } \\
\text { por parceiro íntimo. }\end{array}$ & $\begin{array}{l}\text { Identificar a associação entre } \\
\text { violência por parceiro íntimo e } \\
\text { indicativo de ideação suicida } \\
\text { durante a atual gestação. }\end{array}$ & $\begin{array}{c}\text { Observacional e } \\
\text { transversal. }\end{array}$ & $4^{*}$ \\
\hline $\mathrm{A} 24^{31}$ & $\begin{array}{l}\text { Fatores associados à } \\
\text { violência física por } \\
\text { parceiro íntimo em } \\
\text { usuárias de serviços de } \\
\text { saúde. }\end{array}$ & $\begin{array}{l}\text { Estimar a prevalência de violência } \\
\text { por parceiro íntimo contra mulheres } \\
\text { e identificar fatores associados. }\end{array}$ & Transversal. & $4^{*}$ \\
\hline $\mathrm{A} 25^{32}$ & $\begin{array}{l}\text { Violência entre parceiros } \\
\text { intimos e consumo de } \\
\text { álcool. }\end{array}$ & $\begin{array}{l}\text { Estimar a prevalência de violência } \\
\text { por parceiros íntimos e o consumo } \\
\text { de álcool durante os eventos dessa } \\
\text { violência. }\end{array}$ & Transversal. & $4^{*}$ \\
\hline $\mathrm{A} 26^{33}$ & $\begin{array}{l}\text { Coocorrência de violência } \\
\text { física conjugal e contra } \\
\text { filhos em serviços de } \\
\text { saúde. }\end{array}$ & $\begin{array}{l}\text { Apresentar um perfil de ocorrência } \\
\text { e coocorrência de violência física } \\
\text { conjugal e contra filhos em uma } \\
\text { população atendida em serviço } \\
\text { de saúde, segundo diferentes } \\
\text { características socioeconômicas e } \\
\text { demográficas. }\end{array}$ & Transversal. & $4^{*}$ \\
\hline
\end{tabular}

Nota: ID - identificação; NE - nível de evidência; *questão de significado/experiência.

Fonte: dados da pesquisa.

Referente à caracterização dos estudos, identificou-se um (3,8\%) artigo sobre VCM nos anos de 2003, 2008, 
2011, 2012, 2013, 2014, 2016, 2019 e 2020. Esse número passou para dois $(7,6 \%)$ em 2005, 2009, 2010 e 2018 e, a seguir, nos anos de 2006, 2015 e 2017 , emergiram três $(11,5 \%)$. Referente ao método de pesquisa, predominou a abordagem quantitativa $(76,9 \%, n=20)$, seguida da qualitativa $(23 \%, n=6)$.

A Atenção Primária à Saúde $(26,9 \%, n=7)$ e os hospitais $(26,9 \%, \mathrm{n}=7)$ prevaleceram como cenários de estudo. Também foram encontradas pesquisas em centros urbanos e rurais $(11,5 \%, n=3)$, centros urbanos $(11,5 \%, n=3)$, Delegacias Especializadas no Atendimento à Mulher $(3,8 \%, n=1)$, Centros de Acolhimento a pessoas em situação de rua $(3,8 \%$, $\mathrm{n}=1)$, Complexos Penitenciários $(3,8 \%, \mathrm{n}=1)$, Centros de Referência em Saúde da Mulher (3,8\%, $\mathrm{n}=1$ ), Centros de Referência do HIV/AIDS (3,8\%, $\mathrm{n}=1$ ) e Centro de Atenção Psicossocial Álcool e Drogas $(3,8 \%, \mathrm{n}=1)$. 0s participantes das pesquisas foram mulheres $(76,9 \%, \mathrm{n}=20)$, homens e mulheres $(7,6 \%, n=2)$, gestantes $(7,6 \%, n=2)$, agentes comunitários de saúde $(3,8 \%, n=1)$ e puérperas $(3,8 \%, n=1)$.

Considerando o objetivo do estudo, no que concerne ao perfil da VCM, em relação à idade, preponderou a faixa etária dos 20 aos 40 anos (50\%, $\mathrm{n}=13)$. A maioria se autodeclarou negra, preta ou parda $(26,9 \%, n=7)$. A religião católica predominou $(15,3 \%, n=4)$. A maior parte das vítimas era casada ou residia com parceiro íntimo $(42,3 \%, n=11)$; eram mães ou estavam grávidas $(30,7 \%, \mathrm{n}=8)$; e não realizavam atividade remunerada $(19,2 \%, n=5)$.

Como fatores de risco para a VCM, identificaramse: o uso de drogas e/ou álcool pela vítima ou agressor $(34,6 \%, n=9)$; a mulher ter baixa escolaridade $(50 \%, \mathrm{n}=13)$; e baixa condição socioeconômica $(50 \%, n=13)$. Outro fator importante foi a vítima ter sofrido ou presenciado violência intrafamiliar na infância $(26,9 \%, n=7)$; ter tido iniciação sexual precoce, com menos de 15 anos $(7,69 \%, n=2)$; ter vivido uma infância ausente de brincadeiras e afeto $(3,8 \%, n=1)$; não ter recebido suporte familiar para sair do ciclo de violência $(7,69 \%, n=2)$; e depender financeiramente do agressor $(3,8 \%, n=1)$. Em relação ao agressor foram identificados como principais fatores de risco: ter sofrido agressão na infância $(3,8 \%, \mathrm{n}=1)$; usar álcool e drogas $(30,7 \%$, $\mathrm{n}=8)$; e ter baixa escolaridade $(7,69 \%, \mathrm{n}=2)$.

Considerando que a VCM é um fenômeno mundialmente antigo, que acarreta danos físicos, psicológicos, patrimoniais, sociais e morais à vida das vítimas, várias tipologias foram identificadas, sendo visualizadas em maior prevalência a combinação entre violência física, psicológica e sexual $(61,5 \%, n=16)$; contudo, também foram encontradas isoladamente: violência física $(26,9 \%$, $\mathrm{n}=7)$; violência física e sexual $(3,8 \%, \mathrm{n}=1)$; violência física, sexual e social $(3,8 \%, n=1)$; e violência por parceiro íntimo $(3,8 \%, n=1)$.

Desse modo, as mais prevalentes nos estudos foram: violência física, psicológica e sexual $(65,3 \%, n=17)$; apenas violência física $(26,9 \%$, $\mathrm{n}=7)$; a associação entre violência física, sexual e social $(3,8 \%, n=1)$; e a violência por parceiro íntimo $(3,8 \%, n=1)$. No que diz respeito aos agressores, houve predomínio de parceiros intimos como principais perpetuadores $(50 \%, \mathrm{n}=13)$; seguido de companheiros $(26,9 \%, n=7)$. Companheiros e instituições protetivas apareceram em duas publicações $(7,6 \%)$, não sendo especificado 0 agressor em dois artigos (7,6\%); parceiros íntimos e clientes surgiram em uma produção (3,8\%, $\mathrm{n}=1)$; bem como companheiros, ex-companheiros, desconhecidos e instituições protetivas $(3,8 \%$, $n=1)$.

A violência física prevaleceu como principal forma de agressão. 0 artigo $A 16^{23}$ evidenciou a taxa de prevalência de violência física conjugal por graus de severidade, identificando a ocorrência de violência conjugal grave em $22,1 \%$ dos casos e não grave em $10,5 \%$; enquanto o artigo $\mathrm{A} 9^{16}$ demonstrou prevalência de $36,5 \%$ para essa tipologia. 0s tapas, socos, espancamentos, ameaça/uso de arma e chutes emergiram como as principais formas de agressão.

A violência psicológica foi identificada em associação com outras tipologias. Nesse sentido, pesquisa sinalizou que, para $80,2 \%$ das mulheres, a violência psicológica ocorre, pelo menos, uma vez na vida e, para 50\%, nos últimos 12 meses, sendo mais prevalente o insulto, figurando em $39 \%$ dos casos, seguido por ameaças $(3,8 \%, n=1)$, sendo que em mulheres encarceradas há incidência de $31,8 \%$ nos últimos 12 meses $(3,8 \%, n=1)$.

Diante das situações de VCM, observou-se que as mulheres buscaram atendimentos nos serviços de saúde $(19,2 \%, n=5)^{8,14,16,29,32}$ ou passaram a residir nas ruas e/ou reagiram às agressões $(11,5 \%$, $n=2)^{11,13}$. Contudo, outras medidas também foram observadas, mas em menor frequência, como: as vítimas apresentarem dificuldades em realizar 0 pré-natal completo ${ }^{9}$; apresentaram inadequações no exame citopatológico10; recorreram à polícia, 
benzedeiros, curandeiros, pais de santo, amigos, familiares, centros de saúde, casa de proteção, ambulatórios de saúde mental ${ }^{23}$; procuraram a polícia e atendimentos em serviços de saúde ${ }^{20}$; separaramse do agressor e buscaram atendimentos nos serviços de saúde ${ }^{22}$. Os demais artigos 12,15,17-19,21,24-28,30-31,33 não informaram as medidas adotadas pela vítima.

No que se refere às consequências da violência na vida das mulheres, constatou-se que elas desenvolveram majoritariamente os transtornos mentais. As diferentes produções traziam a combinação dos seguintes transtornos: transtorno de estresse pós-traumático e tentativa de suicídio ${ }^{26}$; transtornos mentais, ideação suicida, insônia acompanhada do uso de ansiolíticos ${ }^{25}$; transtornos mentais e comportamentais, aparecimentos de doenças do trato geniturinário, problemas respiratórios e lesões externas ${ }^{16}$; humor depressivo ansioso, decréscimo da energia vital e sintomas somáticos ${ }^{29}$; ideação suicida ${ }^{30}$; pobreza, experiências de violências, transtornos mentais, dependência de álcool e drogas, falta de amor e rupturas dos vínculos familiares e sociais ${ }^{13}$; a mulher apresentar práticas maternas violentas com seus filhos figurou em uma produção $0^{21}$. 0s demais artigos ${ }^{8-12,14-15,17-20,22-24,27-28,31-33}$ não informaram as consequências da VCM na vida da mulher.

\section{DISCUSSÃO}

Frente aos achados desta revisão, constatou-se a prevalência de estudos no ano de 2017. Ponderase que o aumento de publicações nesse ano possa estar ligado à divulgação da Pesquisa DataSenado ${ }^{34}$ intitulada "Violência Doméstica e Familiar contra a Mulher". Essa produção exibiu o aumento de violência doméstica nos anos de 2015 e 2017, no qual o percentual passou de $18 \%$ para $29 \%$.

Constatou-se predomínio de estudosna Estratégia Saúde da Família. Esse local é o elo de aproximação dos profissionais da saúde com as vítimas de violência ${ }^{35}$, embora a assistência prestada nem sempre seja suficiente e de qualidade ${ }^{36}$, aspectos que podem justificar a realização de pesquisas nesse contexto. Semelhantemente, os hospitais configuraram um local importante para a realização das pesquisas devido aos altos índices de procura nesses serviços por atendimento em função da VCM. Contudo, estudo evidencia a falta de preparo médico para atender a essa demanda, associado à dificuldade na implementação de estratégias efetivas para o enfrentamento desse fenômeno ${ }^{37}$.

A prestação de assistência em saúde inadequada às vítimas é reflexo do modelo biologicista presente na formação profissional que, muitas vezes, limitase somente ao tratamento de lesões físicas, sem abranger o olhar holístico aos indivíduos. Estudo de revisão sobre violência sexual contra as mulheres apontou que os profissionais de saúde apresentam dificuldade em reconhecer e tratar de maneira integral os casos, devido ao despreparo do profissional e do serviço, como também pela lacuna presente na graduação, caracterizada pelo debate incipiente sobre a temática ${ }^{38}$.

Em se tratando do perfil da VCM, estudo coaduna com os achados da revisão em tela, visto que também destacou a maior prevalência $(57,72 \%)$ em mulheres jovens, pretas e pardas $(63,80 \%)$ e gestantes $(12,20 \%)$. Sobre o estado civil, as solteiras apresentavam taxa de $47,64 \%{ }^{39}$, o que diverge dos achados desta revisão, pois preponderaram mulheres casadas ou que conviviam com parceiros íntimos.

A religião católica predominou, corroborando os achados de pesquisa ${ }^{40}$, em que essa religião foi procurada em $25,4 \%$ dos casos de VCM. Outra variável semelhante entre a revisão e essa pesquisa consiste no fato de que muitas mulheres eram mães ou estavam grávidas e ter filhos foi apontado como um dos motivos para as mulheres retornarem ao convívio com o agressor após a violência ${ }^{40}$.

A baixa escolaridade foi um fator condicionante para a VCM. Na mesma direção, estudo identificou que $40,63 \%$ das mulheres possuíam apenas o ensino fundamental ${ }^{39}$. Outro fator predisponente foi a baixa condição socioeconômica e a dependência financeira do agressor, resultado semelhante encontrado em outra produção ${ }^{41}$, na qual apenas $18 \%$ das mulheres exerciam atividade remunerada.

Autores também apontaram o início sexual precoce, antes dos 15 anos, como um fator de risc $0^{42}$. Esse aspecto, muitas vezes, está associado à maior vulnerabilidade, o que também aproxima as adolescentes das situações de VCM ${ }^{43}$.

Ainda se constatou que os parceiros íntimos e companheiros foram os principais agressores. Esse dado coaduna-se com pesquisa realizada sob análise de 130 Autos de Prisão, expondo os maridos/ companheiros como agressores em $61,5 \%$ das situações, seguidos de ex-maridos/companheiros, em $15,4 \% 44$.

No que concerne às tipologias de VCM, estudo 
de revisão detectou predomínio de violência física $(87,5 \%)^{45}$. Essa violência pode ser definida pelas condições em que um indivíduo exerce poder sobre o outro, gerando danos por meio de força física ou $\operatorname{armas}^{46}$.

Outra tipologia verificada foi a violência sexual, que é definida pelos atos ou tentativas sexuais indesejadas e forçadas, praticadas por conhecidos ou não, incluindo os estupros na relação conjugal e em situações de guerra ${ }^{46}$. No Brasil, pesquisa sinaliza o expressivo número de estupros anualmente, correspondendo a $527 \mathrm{mil}$ casos, dos quais apenas $10 \%$ são notificados e $89 \%$ das vítimas são do sexo feminino ${ }^{47}$.

Quanto à violência psicológica, autor lembra que o agressor, antes de agredir fisicamente, reduz a autoestima da mulher, a fim de que ela aceite as agressões ${ }^{48}$. Essa tipologia compreende os atos ou omissões que geram danos emocionais, psicológicos ou de identidade à vítima ${ }^{46}$. Diante disso, autores alertam que uma mesma mulher, no último triênio de sua vida, pode ser vítima de 490 episódios de violência psicológica ${ }^{39}$.

No que se refere ao agressor, produção científica observou que $70,8 \%$ dos homens faziam uso concomitante de substâncias, prevalecendo o uso de álcool em $60 \%$ dos casos, mas também associado a alguma droga em 7,7\%, como maconha, crack e cocaína ${ }^{44}$. Esse achado aproxima-se dos achados do estudo em tela.

Em se tratando das medidas adotadas pelas vítimas, percebeu-se que os resultados da revisão se assemelham aos de um estudo de coorte, no qual as mulheres procuraram instituições religiosas $(25,4 \%)$, serviços de saúde $(27,1 \%)$, delegacias de polícia $(56,6 \%)$, amigos e familiares $(57,6 \%)$. Nessa direção, pondera-se a importância da rede social primária como forma de enfrentamento nos casos de $V_{C M}{ }^{40}$. Com relação às Delegacias de Defesa da Mulher (DDM), em especial, a Política Nacional de Enfrentamento da VCM reforça que esses serviços precisam propor um trabalho articulado em rede, considerando os diferentes níveis de atenção no combate à violência ${ }^{49}$.

Os danos à saúde física e mental causados pela violência são, por vezes, irreversíveis e irreparáveis. Nesse sentido, esta revisão verificou a presença de transtornos mentais devido à violência. Outra revisão indicou que as mulheres vítimas de violência por parceiro íntimo comumente sofrem depressão $(73,7 \%)$ e transtornos de estresse pós-traumático $(52,6 \%)$. Em menor prevalência, também apresentam humor depressivo-ansioso, sintomas somáticos, decréscimo da energia vital e pensamentos depressivos ${ }^{5}$.

As consequências ginecológicas observadas na presente revisão corroboram os resultados de estudo ${ }^{42}$. Nessa pesquisa, identificou-se a ocorrência de contaminação por infecções sexualmente transmissiveis, abortos ilegais, perda da libido, sangramento vaginal, irritação vaginal, infecção no trato urinário e dor pélvica.

Além disso, pode-se observar que, mesmo buscando atendimento em saúde, muitas mulheres acabam indo a óbito. Diante disso, o Mapa da Violência de 2015 exibiu o aumento de 21,0\% de óbitos, entre os anos de 2003 e 2013, sendo que, em 2003, foram contabilizados 3.937 homicídios de mulheres, representando 13 mortes diárias ${ }^{50}$.

\section{CONCLUSÃO}

Ao analisar os resultados desta revisão, é possível traçar o perfil, os fatores de risco e a prevalência da VCM. Com isso, infere-se que os achados podem fornecer subsídios para o enfrentamento desse agravo. Ao mesmo tempo, considera-se que os achados podem contribuir para que os profissionais e estudantes da saúde identifiquem possiveis vítimas de VCM e forneçam uma assistência em saúde mais sensivel e humanizada às suas demandas.

Sendo um fenômeno transcultural, transnacional e que afeta a todas as classes e grupos, a VCM precisa ser reconhecida para que possa ser combatida. Nesse sentido, o combate à violência é longo e árduo. Contudo, a partir de evidências científicas sobre o tema é possível traçar estratégias de enfrentamento e suporte às mulheres. Posto isso, salienta-se a importância da criação e implementação de protocolos e fluxos de atendimento em saúde e sinaliza-se a necessidade de a rede intersetorial estar articulada e agir de maneira conjunta para atender integralmente esse público.

\section{CONTRIBUIÇÃO DAS AUTORAS}

\section{Letícia Barbosa Dias e Lisie Alende Prates} contribuíram com a realização da pesquisa, o delineamento do estudo, a redação e a revisão crítica do manuscrito. Luiza Cremonese contribuiu com a redação e a revisão crítica do manuscrito. 


\section{REFERÊNCIAS}

1. World Health Organization. World report on violence and health. Geneva: World Health Organization; 2002 .

2. Silva EB, Padoin SMM, Vianna LAC. Mulher em situação de violência: limites da assistência. Ciênc Saúde Colet [serial on the internet]. 2015 [cited 2020 Aug 20];20(1):249-58. Available from: https://www.scielo.br/pdf/csc/v20n1/pt 14138123-csc-20-01-00249.pdf

3. Lucena KDT, Deininger LSC, Coelho HFC, Monteiro ACC, Vianna RPT, Nascimento JA. Análise do ciclo da violência doméstica contra a mulher. J Hum Growth Dev [serial on the internet]. 2016 [cited 2020 Aug 20];26(2):13946. Available from: http://pepsic.bvsalud.org/ $\mathrm{pdf} / \mathrm{rbcdh} / \mathrm{v} 26 \mathrm{n} 2 / \mathrm{pt}$ 03.pdf

4. Brasil. Impacto da violência na saúde dos brasileiros. Brasília (DF): Ministério da Saúde; 2005.

5. Santos AG, Monteiro CFS, Feitosa CDA, Veloso C, Nogueira LT, Andrade EMLR. Tipos de transtornos mentais não psicóticos em mulheres adultas violentadas por parceiro íntimo: uma revisão integrativa. Rev EsC Enferm USP [serial on the internet]. 2018 [cited 2020 Aug 20];52: e03328. Available from: https://www.scielo.br/pdf/reeusp/ v52/0080-6234-reeusp-52-e03328.pdf

6. Brasil. Agenda de Prioridades de Pesquisa do Ministério da Saúde. Brasília (DF): Ministério da Saúde; 2018.

7. Paula CC, Padoin SMM, Galvão CM. Revisão integrativa como ferramenta para tomada de decisão na prática em saúde. In: Lacerda MR, Costenaro RGS, editores. Metodologia da pesquisa para enfermagem e saúde: da teoria à prática. Porto Alegre: Moriá; 2015.

8. Hesler LZ, Costa MC, Resta DG, Colomé ICS. Violência contra as mulheres na perspectiva dos agentes comunitários de saúde. Rev gaúch enferm [serial on the internet]. 2013 [cited 2021 June 15];34(1):180-6. Available from: https:// www.scielo.br/j/rgenf/a/MWVc63psXP36k7 CKSP4YSzp/?format $=p d f \&$ lang $=p t$

9. Audi CAF, Segall-Corrêa AM, Santiago SM, Andrade MGG, Pérez-Escamilla R. Violência doméstica na gravidez: prevalência e fatores associados. Rev saúde pública [serial on the internet]. 2008 [cited 2021 June 15];42(5):877-85. Available from: https://www.scielo.br/j/rsp/a/QvwTF5 QSg4JGJmwL9T8rZbL/?format=pdf\&lang=pt

10. Rafael RMR, Moura ATMS. Violência física grave entre parceiros íntimos como fator de risco para inadequação no rastreio do câncer de colo de útero. Cad saúde pública [serial on the internet]. 2017 [cited 2021 June 15];33(12). Available from: $\quad$ https://www.scielo.br/j/csp/a/vwhTT VYVZPBJGGPLW3WRRjM/?format=pdf\&lang $=p t$

11. Rosa AS, Brêtas ACP. A violência na vida de mulheres em situação de rua na cidade de São Paulo, Brasil. Interface (Botucatu) [serial on the internet]. 2015 [cited 2021 June 15];19(53):27585. Available from: https://www.scielo.br/j/icse/ a/8T6c9LN8dqCzSJRFyypZDbT/? lang=pt\&format $=p d f$

12. Sanchotene IP, De Antoni C, Munhós AAR. MARIA, MARIA: concepções sobre ser mulher em situação de rua. Textos contextos (Porto Alegre) [serial on the internet]. 2019 [cited 2021 June 15];18(1):14660. Available from: https://revistaseletronicas. pucrs.br/ojs/index.php/fass/article/ view/29297/18804

13. Souza MRR, Oliveira JF, Chagas MCG, Carvalho ESS. Gênero, violência e viver na rua: vivências de mulheres que fazem uso problemático de drogas. Rev gaúch enferm [serial on the internet]. 2016 [cited 2021 June 15];37(3):e59876. Available from: https://www.scielo.br/j/rgenf/a/ YwcqKKkVhvySMGk83fbXpGj/?format=pdf\&lang=pt

14. Marinheiro ALV, Vieira EM, Souza L. Prevalência da violência contra a mulher usuária de serviço de saúde. Rev saúde pública [serial on the internet]. 2006 [cited 2021 June 15];40(4):60410. Available from: https://www.scielo.br/j/ rsp/a/4579MXXY4FcHVZYKXD6w9Jv/?format=pdf\&l ang $=p t$

15. Moura LBA, Gandolfi L, Vasconcelos AMN, Pratesi R. Violências contra mulheres por parceiro intimo em área urbana economicamente vulnerável, Brasília, DF. Rev saúde pública [serial on the internet]. 2009 [cited 2021 June 15];43(6):94453. Available from: https://www.scielo.br/j/rsp/ a/S6rv06pZ5CYjSLD5MkXcZhS/?format=pdf\&lang= pt

16. Silva IV. Violência contra mulheres: a experiência de usuárias de um serviço de urgência e emergência de Salvador, Bahia, Brasil. Cad saúde pública [serial on the internet]. 2003 [cited 2021 June 15];19(suppl 2):S263-72. Available from: https://www.scielo.br/j/csp/a/kWB7RdzH5 7VCHxGkDBxbf5w/?format=pdf\&lang $=p t$

17. Ferreira VP, Silva MA, Noronha Neto C, Falbo Neto GH, Chaves CV, Bello RP. Prevalência e fatores associados à violência sofrida em mulheres encarceradas por tráfico de drogas no Estado de Pernambuco, Brasil: um estudo transversal. Ciênc Saúde Colet [serial on the internet]. 2014 [cited 2021 June 15];19(7):2255-64. Available 
from: https://www.scielo.br/j/csc/a/44ZXP5fDq WdYP4XNr5Ys5wt/?lang =pt\&format $=p d f$

18. Kronbauer JFD, Meneghel SN. Perfil da violência de gênero perpetrada por companheiro. Rev saúde pública [serial on the internet]. 2005 [cited 2021 June 15];39(5):695-701. Available from: https:// www.scielo.br/j/rsp/a/spsP4pVmDtSt7N5VGXWY5Wc $\angle$ ?format $=p d f \&$ lang $=p t$

19. Ceccon RF, Meneghel SN. HIV e violência contra mulheres: estudo em município com alta prevalência de Aids no Sul do Brasil. Rev Panam Salud Publica [serial on the internet]. 2015 [cited 2021 June 15];37(4/5):287-92. Available from: http://www.scielosp.org/scielo.php?script=sci a rttext\&pid $=S 1020-49892015000400015$

20. Leite FMC, Amorim MHC, Wehrmeister FC, Gigante DP. Violência contra a mulher em Vitória, Espírito Santo, Brasil. Rev saúde pública [serial on the internet]. 2017 [cited 2021 June 15];51:33. Available from: https://www.scielo. $\mathrm{br} / \mathrm{j} / \mathrm{rsp} / \mathrm{a} / \mathrm{FgqPNLYMTBgfvZMHK8zbT \times w/ \text {?format } = \mathrm { p }}$ df\&lang $=p t$

21. Silva JMM, Lima MC, Ludermir AB. Intimate partner violence and maternal educational practice. Rev saúde pública [serial on the internet]. 2017 [cited 2021 June 15];51(34). Available from: https://www.scielo.br/j/rsp/a/jxKW4rFWBCJRY3cZ XCV85Ps/?format $=p d f \&$ lang $=p t$

22. Miranda MPM, de Paula CS, Bordin IA. Violência conjugal física contra a mulher na vida: prevalência e impacto imediato na saúde, trabalho e familia. Rev Panam Salud Publica [serial on the internet]. 2010 [cited 2021 June 15];27(4):3008. Available from: https://www.scielosp.org/ article/rpsp/2010.v27n4/300-308/\#ModalArticles

23. Bruschi A, Paula CS, Bordin IAS. Prevalência e procura de ajuda na violência conjugal física ao longo da vida. Rev saúde pública [serial on the internet]. 2006 [cited 2021 June 15];40(2):25664. Available from: https://www.scielo.br/j/rsp/a/ $\underline{\text { SRg6Lps7G87 } 553 n M G S 8 f r J v / ? \text { format }=p d f \& l a n g=p t}$

24. Oliveira JB, Lima MCP, Simão MO, Cavariani MB, Tucci AM, Florence KC. Violência entre parceiros intimos e álcool: prevalência e fatores associados. Rev Panam Salud Publica [serial on the internet]. 2009 [cited 2021 June 15];26(6):494-501. Available from: http://www.scielosp.org/scielo . $p h p$ ? script $=s c i$ arttext \& pid $=$ S1020-49892009001200004

25. Adeodato VG, Carvalho RR, Siqueira VR, Souza FGM. Qualidade de vida e depressão em mulheres vítimas de seus parceiros Rev saúde pública [serial on the internet]. 2005 [cited 2021 June 15];39(1):108-13. Available from: https:// www.scielo.br/j/rsp/a/kbLB4v3hdrn3fCvDfrk $\mathrm{v} 3 \mathrm{Hx} /$ ? format $=\mathrm{pdf} \&$ lang $=\mathrm{pt}$
26. Vasconcelos Neto PJA, Moreira RS, Oliveira Júnior FJM, Ludermir AB. Tentativa de suicídio, transtorno de estresse pós-traumático e fatores associados em mulheres do Recife. Rev bras epidemiol [serial on the internet]. 2020 [cited 2021 June 15];23:e200010. Available from: https:// www.scielo.br/j/rbepid/a/RLhTKKbDZgWR9Z9Tgs9Lt $\mathrm{gr} /$ ? lang = pt\&format $=\mathrm{pdf}$

27. Rodríguez-Borrego MA， Vaquero-Abellán $M$, Rosa LB. Estudo transversal sobre fatores de risco de violência por parceiro íntimo entre enfermeiras. Rev latinoam enferm [serial on the internet]. 2012 [cited 2021 June 15];20(1):118. Available from: https://www.scielo.br $\mathrm{Lj} / \mathrm{rlae} / \mathrm{a} /$ VxMmVGLsRLxG3kXZZ6bqT3g/?lang=pt\&f ormat $=p d f$

28. Marcacine K0. Intimate partner violence among postpartum women: associated factors. Rev bras enferm [serial on the internet]. 2018 [cited 2021 June 15];71(suppl 3):1306-12. Available from: https://www.scielo.br/j/reben/a/cQWr9dJtpfytMv $\underline{\text { YsNHMcTxw/? } \text { lang }=\text { en \& format }=p d f}$

29. Santos AG, Monteiro CFS. Domains of common mental disorders in women reporting intimate partner violence. Rev latinoam enferm (Online) [serial on the internet]. 2018 [cited 2021 June 15];26:e3099. Available from: https://www. scielo.br/j/rlae/a/fcFq3MbHTWVNSYMFVKgMBWg/ ?format $=p d f \&$ lang $=e n$

30. Fonseca-Machado M0, Alves LC, Haas VJ, Monteiro JCS, Gomes-Sponholz F. Sob a sombra da maternidade: gravidez, ideação suicida e violência por parceiro íntimo. Rev Panam Salud Publica [serial on the internet]. 2015 [cited 2021 June 15];37(4/5):258-64. Available from: https://iris. paho.org/bitstream/handle/10665.2/8376/v37n45a11.pdf? sequence $=1$ \&is Allowed $=y$

31. Vieira EM, Perdona GSC, Santos MA. Fatores associados à violência física por parceiro íntimo em usuárias de serviços de saúde. Rev saúde pública [serial on the internet]. 2011 [cited 2021 June 15];45(4):730-7. Available from: https://www. scielo.br/j/rsp/a/5938GC5pywcKDy7X5K4GGgF/? format $=p d f \&$ lang $=p t$

32. Zaleski M, Pinsky I, Laranjeira R, RamisettyMikler S, Caetano R. Violência entre parceiros íntimos e consumo de álcool. Rev saúde pública [serial on the internet]. 2010 [cited 2021 June 15];44(1):53-9. Available from: https://www. scielo.br/j/rsp/a/dPPY6gJNmncWf4bMXbHX5Ky/? format $=p d f \&$ lang $=p t$

33. Reichenheim ME, Dias AS, Moraes CL. Coocorrência de violência física conjugal e contra filhos em serviços de saúde. Rev saúde pública [serial on the internet]. 2006 [cited 2021 June 15];40(4):595-603. Available from: https:// www.scielo.br/j/rsp/a/Vf5wS5YMrJ5RCpfBJ RrGrhG/?lang=pt\&format $=p d f$ 
34. Instituto de Pesquisa DataSenado. Violência doméstica e familiar contra a mulher. Brasília (DF): Senado Federal; 2017.

35. Oliveira MT, Samico I, Ishigami ABM, Nascimento RMM. Violência intrafamiliar: a experiência dos profissionais de saúde nas Unidades de Saúde da Família de São Joaquim do Monte, Pernambuco. Rev bras epidemiol [serial on the internet]. 2012 [cited 2020 Sept 12];15(1):166-78. Available from: https://www.scielo.br/pdf/rbepid/v15n1/15.pdf

36. Rodrigues EAS, Tavares R, Melo VH, Silva JM, Melo EM. Violência e Atenção Primária à Saúde: percepções e vivências de profissionais e usuários. Saúde debate [serial on the internet]. 2018 [cited 2020 Sept 12];42(n. esp.):55-66. Available from: https://www.scielosp.org/article/ssm/ content/raw/?resource ssm $\mathrm{path}=/ \mathrm{media} / \mathrm{assets} /$ sdeb/v42nspe4/0103-1104-sdeb-42-spe04-0055.pdf

37. Pedrosa CM, Spink MJP. A violência contra mulher no cotidiano dos serviços de saúde: desafios para a formação médica. Saúde Soc [serial on the internet]. 2011 [cited 2021 Jan 06];20(1):124-35. Available from: https://www.scielo.br/pdf/sausoc/ v20n1/15.pdf

38. Aguiar FAR, Dourado JVL, Cavalcanti LF, Vieira LJES, Ferreira Júnior ARF, Silva RM, et al. Formação profissional e atenção a mulher vítima de violência sexual: revisão integrativa. Sanare (Sobral, Online) [serial on the internet]. 2020 [cited 2021 Jan 06];19(2):57-68. Available from: https://sanare. emnuvens.com.br/sanare/article/view/1476/732

39. Oliveira CAB, Alencar LN, Cardena RR, Moreira KFA, Pereira PPS, Fernandes DER. Perfil da vítima e características da violência contra a mulher no estado de Rondônia - Brasil. Rev cuid [serial on the internet]. 2019 [cited 2020 Sept 22];10(1). Available from: http://www.scielo.org.co /pdf/cuid/v10n1/2346-3414-cuid-10-1-e573.pdf

40. Silva RA, Araujo TVB, Valongueiro S, Ludermir $A B$. Enfrentamento da violência infligida pelo parceiro íntimo por mulheres em área urbana da região Nordeste do Brasil. Rev saúde pública [serial on the internet]. 2012 [cited 2020 Sept 18];46(6):1014-22. Available from: https://www. scielo.br/pdf/rsp/v46n6/a03594.pdf

41. Viana AL, Lira MOSC, Vieira MCA, Sarmento SS, Souza APL. Violência contra a mulher. Rev enferm UFPE on line [serial on the internet]. 2018 [cited 2020 Sept 18];12(4):923-9. Available from: https:// periodicos.ufpe.br/revistas/revistaenfermagem/ article/view/110273/28639

42. Baigorria J, Warmling D, Neves CM, Delziovo CR, Coelho EBS. Prevalência e fatores associados da violência sexual contra a mulher: revisão sistemática. Rev salud pública [serial on the internet]. 2017 [cited 2020 Sept 22];19(6).
Available from: https://www.scielosp.org/pdf /rsap/2017.v19n6/818-826/pt

43. Taquette SR, Vilhena MM. Uma contribuição ao entendimento da iniciação sexual feminina na adolescência. Psicol estud [serial on the internet]. 2008 [cited 2020 Sept 22];13(1):105-14. Available from: https://scielo.br/pdf/pe/v13n1/v13n1a12. $\underline{p d f}$

44. Madureira AB, Raimondo ML, Ferraz MIR, Marcovicz GV, Labronici LM, Mantovani MF. Perfil de homens autores de violência contra mulheres detidos em flagrante: contribuições para o enfrentamento. Esc Anna Nery Rev Enferm [serial on the internet]. 2014 [cited 2020 Sept 20];18(4):600-6. Available from: http://www.revistaenfermagem.eean.edu.br/2017/ detalhe artigo.asp?id $=1240$

45. Duarte MC, Fonseca RM, Souza V, Pena ED. Gender and violence against women in nursing literature: a review. Rev bras enferm [serial on the internet]. 2015 [cited 2020 Sept 20];68(2):325-32. Available from: https://www.scielo.br/pdf/reben/ v68n2/en 0034-7167-reben-68-02-0325.pdf

46. Brasil. Violência intrafamiliar: orientações para prática em serviço. Brasília (DF): Ministério da Saúde; 2001.

47. Cerqueira D, Coelho DSC. Estupro no Brasil: uma radiografia segundo os dados da Saúde (versão preliminar). Brasília (DF): Instituto de Pesquisa Econômica Aplicada; 2014.

48. Miller L. Protegendo as mulheres da violência doméstica. Seminário de treinamento para juízes, procuradores, promotores e advogados no Brasil. $2^{\mathrm{a}}$ ed. Brasília (DF): Tahirid Justice Center; 2002.

49. Machado DF, Almeida MAS, Dias A, Bernardes JM, Castanheira ERL. Violência contra a mulher: o que acontece quando a Delegacia de Defesa da Mulher está fechada? Ciênc Saude Colet [serial on the internet]. 2020 [cited 2020 0ct 15];25(2):48394. Available from: https://www.scielo.br/ $\mathrm{pdf} / \mathrm{csc} / \mathrm{v} 25 \mathrm{n} 2 / 1413-8123-\mathrm{csc}-25-02-0483 . \mathrm{pdf}$

50. Waiselfisz JJ. Mapa da Violência 2015: Homicídio de mulheres no Brasil. Brasília (DF): Flacso; 2015.
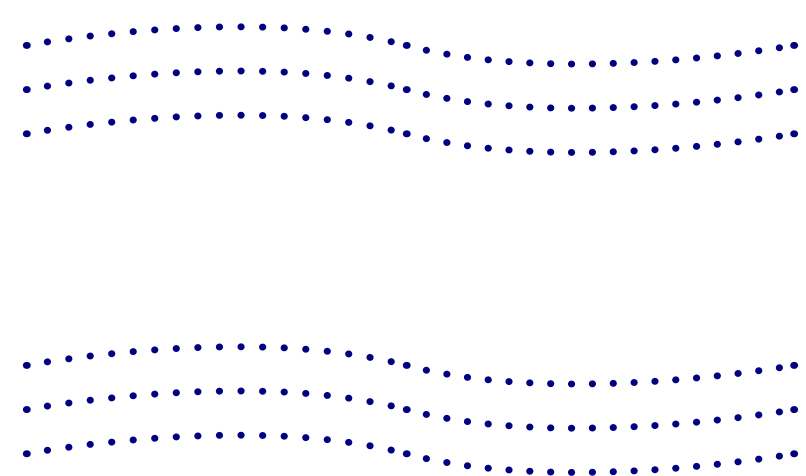